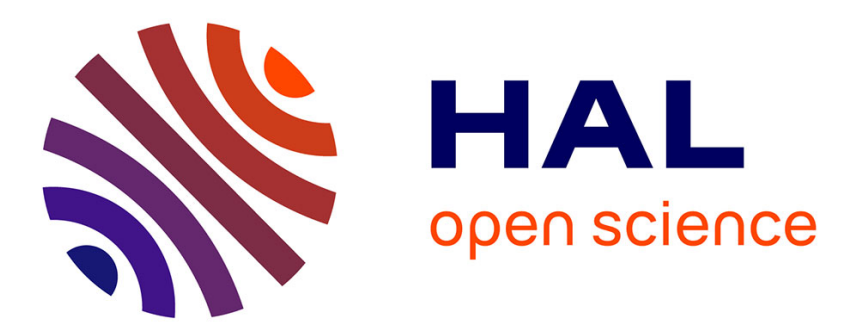

\title{
X-ray Scattering Determination of the Structure of Water during Carbon Nanotube Filling
}

Erwan Paineau, Pierre-Antoine Albouy, Stéphan Rouzière, Andrea Orecchini, Stéphane Rols, Pascale Launois

\section{To cite this version:}

Erwan Paineau, Pierre-Antoine Albouy, Stéphan Rouzière, Andrea Orecchini, Stéphane Rols, et al.. X-ray Scattering Determination of the Structure of Water during Carbon Nanotube Filling. Nano Letters, 2013, 13 (4), pp.1751 - 1756. 10.1021/nl400331p . hal-03330275

\section{HAL Id: hal-03330275 https://hal.science/hal-03330275}

Submitted on 31 Aug 2021

HAL is a multi-disciplinary open access archive for the deposit and dissemination of scientific research documents, whether they are published or not. The documents may come from teaching and research institutions in France or abroad, or from public or private research centers.
L'archive ouverte pluridisciplinaire HAL, est destinée au dépôt et à la diffusion de documents scientifiques de niveau recherche, publiés ou non, émanant des établissements d'enseignement et de recherche français ou étrangers, des laboratoires publics ou privés. 


\section{X-ray Scattering Determination of the Structure of Water during Carbon Nanotube Filling}

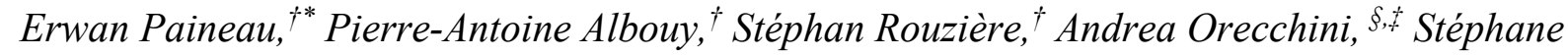
Rols, ${ }^{\S}$ Pascale Launois ${ }^{\dagger^{*}}$

† Laboratoire de Physique des Solides, UMR 8502, Université Paris-Sud, Bâtiment 510, 91405

Orsay Cedex, France

$\S$ Institut Laue-Langevin, BP 156, 38042 Grenoble, France

† Dipartimento di Fisica, Università di Perugia, I-06123 Perugia, Italy 


\begin{abstract}
We present in-situ monitoring of water filling of single-walled carbon nanotubes at room temperature, using X-ray scattering (XRS). A systematic method is developed to determine the water radial density profile. Water filling is homogeneous below about $5 \%$ in mass, whereas it structures into three layers above. These results should motivate further theoretical and simulations studies and allow getting a better understanding of the very peculiar properties of water confined in hydrophobic environment.
\end{abstract}

KEYWORDS: Carbon nanotube, water, confinement, adsorption, X-ray scattering, radial structure.

Figure for TOC only
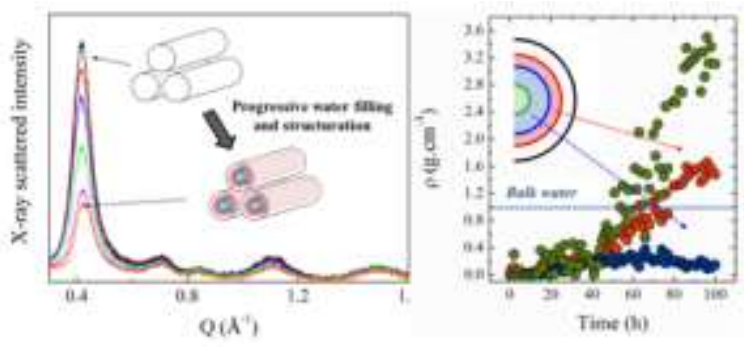
Carbon nanotubes (CNT) are ultimate one-dimensional channels for nanofluidics, mimicking well-known biological systems such as aquaporins or ion-channels ${ }^{1-2}$ and offering potential applications in water desalination membranes or molecular gatekeeper devices. ${ }^{3-5}$ The seminal paper of Hummer et al, ${ }^{6}$ based on molecular dynamics (MD) simulations, reported the spontaneous filling by water of sub-nanometer single walled carbon nanotubes (SWCNT) and fast conduction of water through SWCNT. The water-nanotube interfacial friction is expected to exhibit a strong curvature dependence, increasing with the nanotube diameter. ${ }^{7}$ Enhanced water flow was measured in 2 to $7 \mathrm{~nm}$ diameter nanotubes ${ }^{8-9}$ and even in larger carbon nanopipes, ${ }^{10}$ which is therefore promising in regard to the smallest diameter nanotubes. Thermodynamical properties of water confined in SWCNTs are also most exciting, with predicted and observed new ice structures of ordered $n$-gonal nanotubes, ${ }^{11-14}$ peculiar wet-dry phase transitions with lowering temperature, ${ }^{15}$ and anomalous soft dynamics. ${ }^{16}$

Filling of hydrophobic carbon nanotubes with water, at the basis of all properties discussed above, is a counterintuitive phenomenon. Nevertheless it does occur spontaneously when open carbon nanotubes are soaked in liquid water ${ }^{17-19}$ as well as when they are exposed to water gas, ${ }^{13,16,20}$ as shown by various experimental methods, such as X-ray and neutron scattering, Raman and photo-luminescence spectroscopies and nuclear magnetic resonance. Modification of nanotube densities due to their spontaneous filling by water is in fact a key parameter to take into account to explain separation of SWCNT by gradient centrifugation..$^{21-22}$ Based on numerical simulations, spontaneous filling of carbon nanotubes has been reported from the liquid phase, ${ }^{6,23-24}$ as well as from the gas phase. ${ }^{25}$ Pascal and co-workers ${ }^{24}$ underlined that water presents specific tetrahedral H-bonding in the bulk, so that its confinement, and the subsequent loss of tetrahedral bonding, can lead to an entropy gain. Modification of the H- 
bonding of water confined in CNT was confirmed by an experimental vibrational spectroscopy study. ${ }^{26}$ Moreover, MD calculations of the radial water density profiles highlight structuring phenomena from a single-file mode to a layered mode with increasing diameter, with specific $\mathrm{H}-$ bonding in the different modes. ${ }^{23,25,27-29}$ Water structure during SWCNT filling, depending of the filling rate, is a key parameter to understand confined water thermodynamics, SWCNT-water interactions, and thus the original properties of water confined in SWCNT., ${ }^{6,29}$ NMR measurements of adsorption-desorption curves have been performed by Mao and co-workers ${ }^{20}$ but, to the best of our knowledge, the structure of water during nanotube filling has not been studied experimentally.

In this Letter, we present the first X-ray scattering (XRS) analysis of the progressive filling of SWCNT by water. Oxygen and carbon being quite similar X-ray scatterers, XRS is well suited for studying the filling of carbon nanotubes by water, thanks to strong interference effects. Experiments were carried out on a powder of open SWCNT (MER Corp., USA) with a mean diameter of $14.1 \AA$. The powder was packed in an unsealed glass capillary in a home-made humidity chamber specially designed for in-situ X-ray scattering experiments. It can be either pumped under primary vacuum at different temperatures, or placed under saturating vapor pressure of water. X-ray scattering measurements were performed at a wavelength of $1.5418 \AA$. Experimental details are provided in the Supporting Information (S.I.). Experiments were performed on three different samples and led to similar conclusions.

SWCNT samples were first heated at $333 \mathrm{~K}$ under vacuum to remove air adsorbed before the experiment ${ }^{30}$ and they were then cooled down to $298 \mathrm{~K}$. The corresponding XRS diagram is shown in Figure 1 (black curve). 


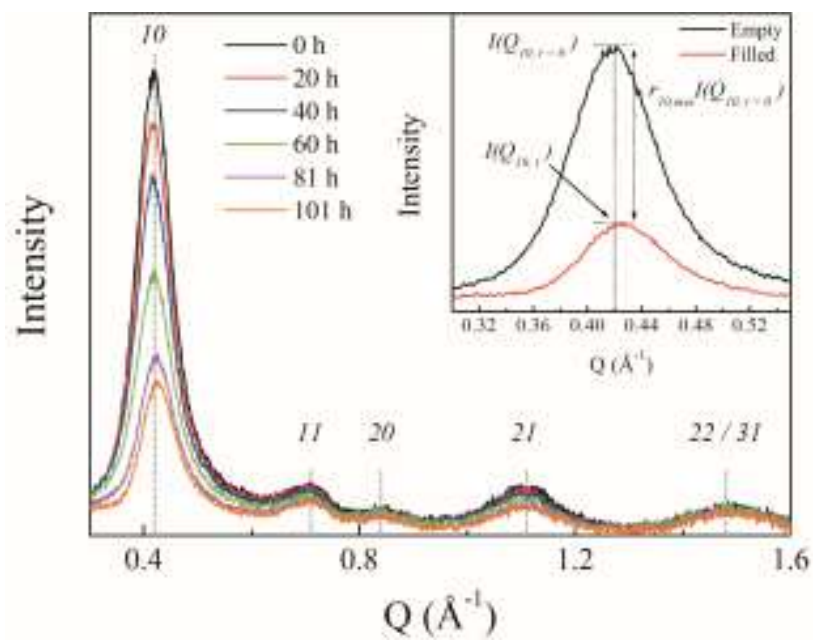

Figure 1. Room temperature XRS diagrams of SWCNT during water adsorption, under saturated vapor pressure (exposure time $=1$ hour). The black curve corresponds to the empty state $(t=0)$. The hk indices refer to the positions of the $2 \mathrm{D}$ hexagonal reciprocal lattice peaks of SWCNT bundles. The inset illustrates the definition of the intensity ratio $r_{h k, \exp }(t)$ for the 10 peak, at $\mathrm{Q}_{10}=$ $0.42 \AA^{-1}$, between the empty $I\left(Q_{10, t=0}\right)$ (black curve) and the completely filled $I\left(Q_{10, t=101} h\right)$ (red curve) states, respectively.

Nanotubes are organized in small bundles on a 2-dimensional (2D) hexagonal lattice, so the XRS diagram presents broad peaks around hk reciprocal positions. ${ }^{31}$ The formalism developed by our team to fit the XRS data ${ }^{32-34}$ is detailed in SI. In the studied Q-range, a carbon nanotube can be considered as a homogeneous cylinder and intensities are modulated by the square of the Fourier transform $F_{\mathrm{CNT}}$ of the nanotube density projected along its axis:

$$
F_{C N T}(Q)=2 \pi \sigma_{C} f_{C}(Q) r_{\text {tube }} J_{0}\left(Q r_{\text {tube }}\right)
$$

where $J_{0}$ is the zero-order cylindrical Bessel function, $r_{\text {tube }}$ the tube radius, $\sigma_{C}$ the carbon atomic density on the nanotube surface $\left(\sigma_{C}=0.37\right.$ atoms. $\left.\AA^{-2}\right)$ and $f_{c}$ the carbon scattering factor. The best fit of experimental data for the empty SWCNT powder is obtained with $r_{\text {tube }}=7.05 \pm 1 \AA$ and for about 30 tubes/bundle (Figure S1). 
Let us now consider the evolution of the XRS diagrams during the filling of SWCNT by water. Empty nanotubes were exposed to saturated vapor pressure of water, at room temperature. XRS diagrams were recorded as a function of time, starting from the beginning of exposure at $t=$ 0 . Contrary to previous studies where samples were water-filled beforehand, ${ }^{13-16}$ we registered XRS diagrams in-situ, whilst the sample was exposed to water gas at constant temperature. Typical diagrams are shown in Figure 1. Filling with water took about 100 hours for the studied sample. However, the complete filling time was found to vary between 20 min and 100 hours depending on experimental conditions such as powder density in the capillary. We underline that time is not the relevant parameter in our study, which is focused on the structure of water during nanotube filling. We observe a progressive decrease of the intensity of the 10 peak. It is known that fully water-filled nanotubes present a much weaker 10 peak than empty ones. ${ }^{13}$ The progressive intensity decrease is thus related to progressive water filling of the nanotube. Hypothesis of intertube filling by water (Figure S2) can be ruled out because it would induce an increase of the 10 peak intensity, ${ }^{13}$ as is discussed in S.I. and shown in Figure S3.

To go further in data analysis, we consider two different structural models for water inside nanotubes. The simplest model (model 1) assumes a homogeneous distribution of water inside the nanotube (Figure 2a). The radius $r_{l}$ of the water cylinder is defined from that of the nanotube, $r_{\text {tube }}$, by $r_{l}=r_{\text {tube }}-2.7 \AA$, to preserve a minimum distance between oxygen and carbon atoms..$^{21,23}$ It can be pointed out that X-rays interact with electrons so that hydrogen atoms are nearly invisible compared to oxygen ones. As mentioned in the introduction, MD simulations give access to water density profiles. ${ }^{23}$ For filled nanotubes with diameter around $14 \AA$, a layered structure is evidenced, with higher water densities at the center of the tube and in a larger-radius cylindrical layer (see Figure 9 in ref. [23]). It does not depend on nanotubes helicities but only on 
their diameters. ${ }^{35} \mathrm{We}$ approximate such layer structure by considering a constant water density $\rho_{3}$ in an internal cylinder of radius $r_{3}$, a different constant density $\rho_{1}$ in a layer defined by radii $r_{1}$ and $r_{2}$, and a third density $\rho_{2}$ in between, as is shown in Figure $2 \mathrm{~b}$ (model 2). To account for minimum carbon-oxygen and oxygen-oxygen distances, as well as for the thickness of the water layer close to the tube wall, one takes $r_{1}=r_{\text {tube }}-2.7 \AA, r_{2}=r_{1}-1 \AA$ and $r_{3}=1 \AA$. As is detailed in S.I., following approaches already developed for peapods ( $\left.\mathrm{C}_{60} @ \mathrm{SWCNT}\right)$ and double-walled carbon nanotubes, ${ }^{33-34}$ one finds that the scattered intensity is now modulated by the square of the projected densities of the nanotube and of water inside:

$$
F(Q)=F_{C N T}(Q)+F_{\text {water }}(Q)
$$

with $F_{\text {water }}(Q)$ for model 1 and model 2 given respectively by equations (3a) and (3b):

$$
\begin{gathered}
F_{\text {water }}(Q)=\frac{2 \pi f_{H_{2} O}}{m_{H_{2} O} Q}\left[\rho_{1} r_{1} J_{1}\left(Q r_{1}\right)\right] \\
F_{\text {water }}(Q)=\frac{2 \pi f_{H_{2} O}}{m_{H_{2} O} Q}\left[\rho_{1} r_{1} J_{1}\left(Q r_{1}\right)+\left(\rho_{2}-\rho_{1}\right) r_{2} J_{1}\left(Q r_{2}\right)+\left(\rho_{3}-\rho_{2}\right) r_{3} J_{1}\left(Q r_{3}\right)\right]
\end{gathered}
$$

$J_{1}$ is the cylindrical Bessel function of order $1, f_{\mathrm{H}_{2} \mathrm{O}}$ is the scattering form factor of water and $m_{\mathrm{H}_{2} \mathrm{O}}=18 u$ is its molecular mass. Typical calculated XRS patterns are drawn in Figure 2c for empty tubes and filled ones, for models 1 and 2 with the same water-to-carbon mass ratio (15.5\% $w t$.$) . Beyond the decrease of the 10$ peak intensity, consistent with a filling of the inner cavity, the intensity of the others peaks are differently affected depending upon which model is considered and precise information on the radial structure of water may be thus accessed. 

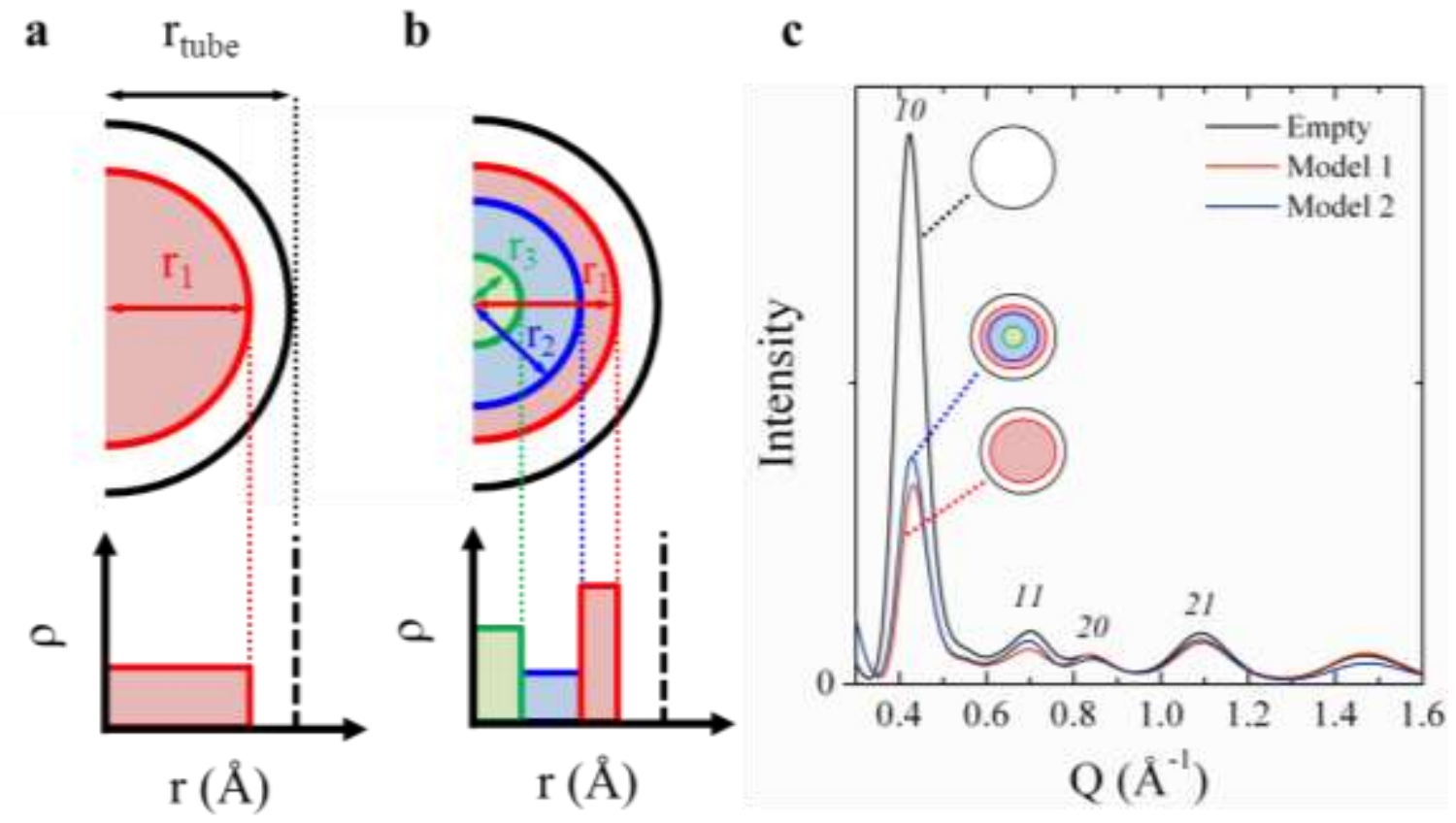

Figure 2. Representation of radial density profiles of water used to simulate the XRS diagrams of SWCNT during water filling: (a) Model 1; (b) Model 2. (c) Simulated XRS diagrams calculated for $r_{\text {tube }}=7.05 \pm 1 \AA, r_{\text {tube }}$ being the nanotube radius, and $N=31$ tubes/bundle. Black curve: empty tubes. Red and blue curves correspond to water structure models 1 and 2,

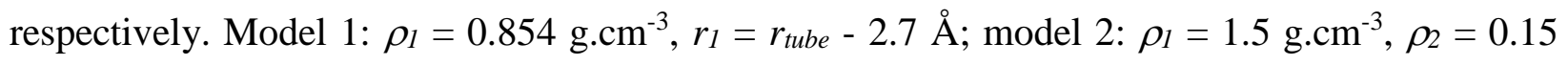
g.cm ${ }^{-3}, \rho_{3}=3.2$ g.cm ${ }^{-3}, r_{1}=r_{\text {tube }}-2.7 \AA, r_{2}=\mathrm{r}_{1}-1 \AA, \mathrm{r}_{3}=1 \AA$.

We propose in this letter a simple and systematic method to determine the water radial density profile from XRS data. Using the experimental XRS diagram obtained at $t=0$ as our internal reference for empty tubes, we define $r_{h k, \exp }($ see inset in Figure 1b) as:

$$
r_{h k, \exp }=\frac{I\left(Q_{h k, t}\right)-I\left(Q_{h k, t=0}\right)}{I\left(Q_{10, t=0}\right)}
$$


where the hk indices refer to hexagonal lattice peaks 10,11, 20 and 21, with $Q_{h k}=0.42$, $0.71,0.84$ and $1.11 \AA^{-1}$, respectively. The normalization to the value of the most intense peak, the 10 one at $t=0$, allows us to minimize the background contribution. Experimental intensity ratios $r_{h k, e x p}$ are drawn as a function of time in Figure 3. As visible in Figure 1b, the intensities of the $10,11,20$ and 21 peaks decrease with time of exposure to water saturated vapor pressure. The representation used here allows us to clearly identify some subtle variations. Indeed, while the intensity ratios $r_{h k, \text { exp }}$ relative to the 10,11 and 21 peaks drop continuously, that of the 20 peak remains constant at first and then drops by a factor two times smaller than the 11 and 21 peaks. After sufficient time, all ratios become constant, which shows that SWCNTs are fully filled by water. Data in Figure 3 correspond to the sample with the longer filling time, for which every single X-ray measurement lasted about one hour and therefore presents the best statistics. We underline however that the experimental curves $r_{h k, \text { exp }}$ obtained from the three different samples studied do superimpose after ad-hoc time renormalisation.
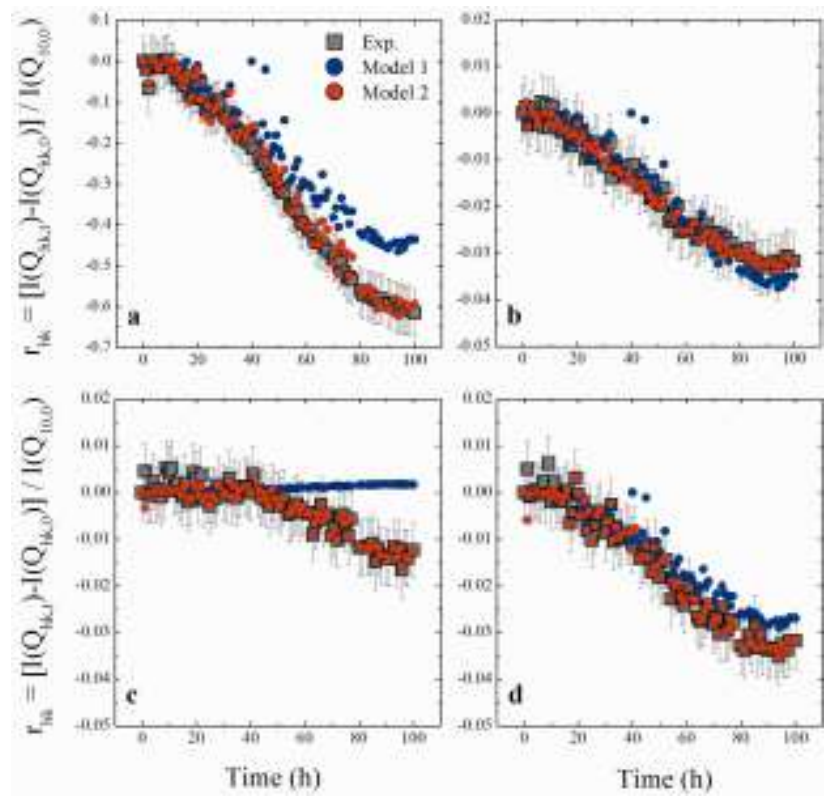
Figure 3. Evolution of the experimental $r_{h k, \exp }$ (grey squares) and calculated $r_{h k, \text { calc }}$ intensity ratios for the (a) 10; (b) 11; (c) 20 and (d) 21 peaks, as a function of time, using model 1 (blue circles) and model 2 (red circles)

Calculations of scattered intensities and thus of the corresponding ratios $r_{h k, c a l c}$ were performed for models 1 et 2 . By minimizing the sum of the square of the differences between calculated and measured ratios, we obtain optimal values of the model parameters ( $\rho_{l}$ for model $1 ; \rho_{l}, \rho_{2}$ and $\rho_{3}$ for model 2). Corresponding $r_{h k, c a l c}$ ratios are reported on Figure 3. The simpler model of a unique water cylinder correctly reproduces experimental values up to $t=40$ hours but it does not allow adjustment for longer times, especially in the case of the 20 peak. In the calculated XRS diagrams, the 20 peak remains constant as is illustrated in Figure 2c. Indeed, within model 1 , the form factor of water (equation (3a)) is close to zero at $Q=0.84 \AA^{-1}$. On the other hand, it is possible to reproduce all $r_{h k, e x p}$ values within model 2. Corresponding values for parameters $\rho_{i}$ are drawn in Figure 4(a).
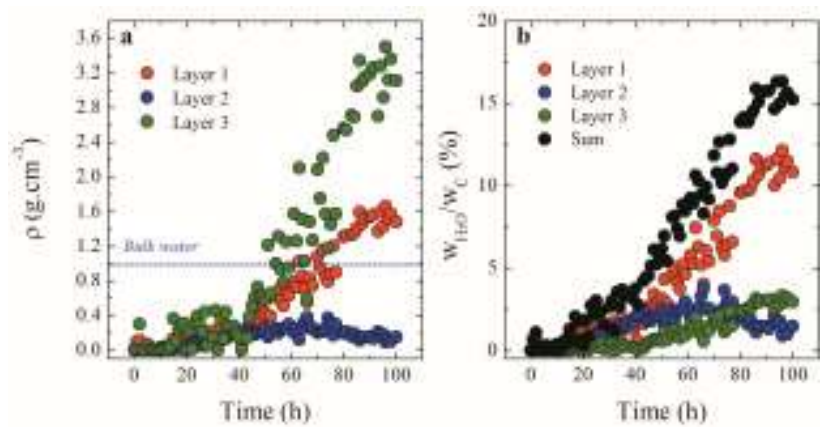

Figure 4. Evolution as a function of time of (a) the water densities and (b) the water weight fractions obtained for the three layers model. Numbers 1 to 3 refer to the layers from the wall towards the center of the SWCNT, respectively.

For times below 40 hours, the density of the three layers are found to be roughly the same, within error bars, which is consistent with the correct fit of the data with a unique cylinder 
(for $\rho_{l}=\rho_{2}=\rho_{3}$, model 2 is equivalent to model 1). After 40 hours, a clear structuration of water in layers is observed, with $\rho_{2}$ smaller than $\rho_{l}$ and $\rho_{3}$. The hypothesis behind our analysis that nanotubes are uniformly filled with water along their length, at any time, seems reasonable considering the fast transport of water inside nanotubes. ${ }^{6-9} \mathrm{We}$ further checked it by considering, in the minimization procedure, for both models 1 and 2, an adjustable proportion of empty tubes or empty parts of tubes, others being fully filled. As expected, no correct agreement with experimental results was found with empty tubes or partially filled tubes, which validates our hypothesis of homogeneous water filling at the time scale of the experiments. It should also be emphasized that the structuration in layers above 40 hours is a robust result. Indeed, we have slightly modified the values of the radii defining the layers extension in model 2 in a realistic manner with respect to the characteristics of the carbon-water and water-water potentials. ${ }^{23}$ The layer structuration always occurs around 40 hours but the value of the internal density $\rho_{3}$ strongly depends on the chosen value of the internal cylinder radius $r_{3}$. It varies in the final state from $3.2 \mathrm{~g} . \mathrm{cm}^{-3}$ for $r_{3}=1 \AA$ to $1.6 \mathrm{~g} . \mathrm{cm}^{-3}$ if $r_{3}=1.55 \AA$, this last value being equal to $r_{2}-1.8 \AA$ for a tube radius of $7.05 \AA$. Projection of the water density in the inner cylinder on the nanotube axis reveals that the mean intermolecular distance $L=m_{H_{2} O} /\left(\rho_{3} \pi r_{3}^{2}\right)$ is equal to $3 \AA$ for $r_{3}=1 \AA$, corresponding to water molecules arranged in a single file mode. ${ }^{6}$

We report in Figure $4 \mathrm{~b}$ the water weight fraction calculated from the density values of Figure $4 \mathrm{a}$, as explained in S.I.. It follows that at complete filling, most of the water molecules (70-80\% of them) are in fact located in the outer cylinder close to the nanotube wall and that uncertainties on the determination of the density $\rho_{3}$ in the inner cylinder have only a minor impact on the total water weight fraction. The total weight fraction of fully filled nanotubes is found to be equal to $15.5 \%$. Note that similar values of 11.3 and $13.6 \%$ are reported by the 
teams of Maniwa and Kolesnikov in refs. [13] and [16], respectively. Larger water weight fractions of 21 and $25 \%$ were further given in refs [14-15]; however, the authors did not discuss the origin of the increase in water uptake with respect to their previous study. ${ }^{13}$ Our result can also be compared with water weight fractions deduced from MD Simulations (Table S1 in S.I.). Calculations lead to weight fractions between 24 and $29 \%{ }^{15,16,21,23-25,27,36}$ A detailed discussion about calculation methods and models of potentials used in the literature is beyond the scope of this letter. Let us only stress that MD simulations were either performed for nanotubes immersed in liquid water, ${ }^{23}$ or for nanotubes exposed to water vapor, ${ }^{25}$ as in our experiments, and that it does not lead to noticeably different densities at complete filling. Calculation of water weight fractions deduced from MD simulations had not been carried out in a systematic way up to now. Discrepancies between calculation results and at least part of experimental results is underlined here for the first time. Moreover, MD simulations mainly considered fully filled nanotubes. The analysis of our XRS diagrams allowed us to determine, for the first time, both the mass fraction and the radial distribution of water in SWCNT during filling. These data should be most useful for future simulations and for improving our knowledge of the water-water and water-carbon interactions in confinement.

Density values obtained in Figure 4a indicate that water filling is rather homogeneous below about $5 \%$ in mass, while it structures into three layers for higher water contents, with a much weaker density in the layer between the external and the internal ones. Striolo and coworkers studied water adsorption on the basis of Monte Carlo simulations. ${ }^{25}$ For $(10,10)$ nanotubes with diameter close to those of the nanotubes studied here, they found that water first adsorbs preferentially close to the nanotube wall, even if it is not in sufficient amount to form a monolayer. We show here experimentally that the first step of nanotube filling does not involve 
the preferential location of water close to the nanotube wall. Indeed, the latter picture would correspond to the condition $\rho_{3}=\rho_{2}=0$, while we measured $\rho_{3} \approx \rho_{2} \approx \rho_{1} \neq 0$ (see also Figure S4).

Pascal and co-workers calculated the entropy and enthalpy components of the free energy of filled nanotubes, depending on their diameter, to determine the driving forces for the filling of carbon nanotubes with water. ${ }^{24}$ Entropy is found to be favorable and dominant in tubes larger than $14 \AA$. They attributed the increase of entropy of confined water, with respect to that of bulk water, to enhanced in-plane translational motions of the molecules. This result is corroborated by our density profiles, where the intermediate layer density is non-zero $\left(\rho_{2} \neq 0\right)$. Within such a picture, this intermediate layer could act as a transition zone between the two layers of higher densities.

In summary, we have presented the first in-situ monitoring of water filling of SWCNT, using X-ray scattering. We observed a progressive decrease of the scattered intensities upon exposure to water vapor. A careful analysis of these variations in intensity allowed us to determine the radial density profile during the filling procedure, as well as the proportion of encapsulated water. Water filling is rather homogeneous below about $5 \%$ in mass, whereas it structures into three layers for higher water contents. Maximum filling corresponds to $\sim 15.5 \%$ in mass of water. These experimental results should motivate further theoretical and simulation studies and allow one to get a better understanding of the very peculiar structure and properties of confined water in hydrophobic environments.

\section{ASSOCIATED CONTENT}

Supporting Information. Details of sample preparation, XRD setup, simulation formalism and additional figures. This material is available free of charge via the Internet at http://pubs.acs.org. 


\title{
AUTHOR INFORMATION
}

\section{Corresponding Authors}

* Emails: $\quad$ erwan-nicolas.paineau@u-psud.fr

\author{
pascale.launois@u-psud.fr
}

\section{ACKNOWLEDGMENT}

The research described here has been supported by Triangle de la physique contract 2010-086.

The authors thank Dr. P. Levitz and Dr. R. Pellenq for fruitful discussions about the results presented in this letter. They also acknowledge D. Petermann, P. Joly and G. Guillier for technical assistance in XRS measurements.

\section{REFERENCES}

(1) Doyle, D. A.; Cabral, J. M.; Pfuetzner, R. A.; Kuo, A.; Gulbis, J. M.; Cohen, S. L.; Chait, B. T.; MacKinnon, R. Science 1998, 280, 69-77.

(2) Murata, K.; Mitsuoka, K.; Hirai, T.; Walz, T.; Agre, P.; Heymann, J. B.; Engel, A.; Fujiyoshi, Y. Nature 2000, 407, 599-605.

(3) Kalra, A.; Garde, S.; Hummer, G. Proc. Natl. Acad. Sci. 2003, 100, 10175-10180.

(4) Corry, B. J. Phys. Chem. B 2008, 112, 1427-1434.

(5) Fornasiero, F.; Park, H. G.; Holt, J. K.; Stadermann, M.; Grigoropoulos, C. P.; Noy, A.; Bakajin, O. Proc. Natl. Acad. Sci. 2008, 105, 17250-17255.

(6) Hummer, G.; Rasaiah, J. C.; Noworyta, J. P. Nature 2001, 414, 188-190.

(7) Falk, K.; Sedlmeier, F.; Joly, L.; Netz, R. R.; Bocquet, L. Nano Lett. 2010, 10, 4067-4073.

(8) Majumder, M.; Chopra, N.; Andrews, R.; Hinds, B. J. Nature 2005, 438, 44-44. 
(9) Holt, J. K.; Park, H. G.; Wang, Y.; Stadermann, M.; Artyukhin, A. B.; Grigoropoulos, C. P.; Noy, A.; Bakajin, O. Science 2006, 312, 1034-1037.

(10) Whitby, M.; Cagnon, L.; Thanou, M.; Quirke, N. Nano Lett. 2008, 8, 2632-2637.

(11) Koga, K.; Gao, G. T.; Tanaka, H.; Zeng, X. C. Nature 2001, 412, 802-805.

(12) Takaiwa, D.; Hatano, I.; Koga, K.; Tanaka, T. Proc. Natl. Acad. Sci. 2008, 105, 39-43.

(13) Maniwa, Y.; Kataura, H.; Abe, M.; Suzuki, S.; Achiba, Y.; Kira, H.; Matsuda, K. J. Phys. Soc. Jpn. 2002, 71, 2863-2866.

(14) Maniwa, Y.; Kataura, H.; Abe, M.; Udaka, A.; Suzuki, S.; Achiba, Y.; Kira, H.; Matsuda, K.; Kadowaki, H.; Okabe, Y. Chem. Phys. Lett. 2005, 401, 534-538.

(15) Kyakuno H.; Matsuda, K.; Yahiro, H.; Inami, Y.; Fukuoka, T.; Miyata, Y.; Yanagi, K.; Maniwa, Y.; Kataura, H.; Saito, T.; Yumura, M.; Iijima, S. J. Chem. Phys. 2011, 134, 244501.

(16) Kolesnikov, A. I.; Zanotti, J. M.; Loong, C. K.; Thiyagarajan, P.; Moravsky, A. P.; Loutfy, R. O.; Burnham, C. J. Phys. Rev. Lett. 2004, 93, 035503.

(17) Sharma S.C.; Singh D.; Li Y. J. Raman Spectrosc. 2005, 36, 755-761.

(18) Chen Q.; Herberg J.; Mogilevsky G.; Wang H.; Stadermann M.; Holt J.; Wu Y. Nano letters, 2008, 8, 1902-1905.

(19) Cambré S.; Santos S.M.; Wenseleers W.; Nugraha A.R.T.; Saito R.; Cognet L.; Lounis B.; ACS Nano, 2012, 6, 2649-2655.

(20) Mao, S.; Kleinhammes, A; Wu, Y. Chemical Physics Letters 2006, 421, 513-517.

(21) Quintillá A.; Hennrich F.; Lebedkin S.; Kappes M.M.; Wenzel W. Phys. Chem. Chem. Phys. 2010, 12, 902-908.

(22) Cambré S.; Wenseleers W. Angew. Chem. 2011, 50, 2761-2768.

(23) Alexiadis ; A. Kassinos, S. Chem. Rev. 2008, 108, 5014-5034 and references therein. 
(24) Pascal, T. A.; Goddard, W. A.; Jung, Y. Proc. Natl. Acad. Sci. 2011, 108, 11794-11798.

(25) Striolo, A.; Chialvo, A. A.; Gubbins, K. E.; Cummings, P. T. J. Chem. Phys. 2005, 122, 234712.

(26) Byl, O.; Liu, J. C.; Wang, Y.; Yim, W. L.; Johnson, J. K.; Yates, J. T. Jr. J. Am. Chem. Soc. 2006, 128, 12090-12097.

(27) Gordillo, M. C.; Martí J. Chem. Phys. Lett. 2000, 329, 341-345.

(28) Hanasaki, I.; Nakatani, A. J. Chem. Phys. 2006, 124, 174714.

(29) Joseph, S.; Aluru, N. R. Nano Lett. 2008, 8, 452-458.

(30) Maniwa Y.; Kumazawa Y.; Saito Y.; Tou H.; Kataura H.; Ishii H.; Suzuki S.; Achiba Y.; Fujiwara A.; Suematsu H. Jpn. J. Appl. Phys. 1999, 38, L668-670

(31) Thess, A.; Lee, R.; Nikolaev, P.; Dai, H.; Petit, P.; Robert, J.; Xu, C.; Lee, Y. H.; Kim, S. G.; Rinzler, A. G.; Colbert, D. T.; Scuseria, G. E.; Tomanek, D.; Fischer, J. E.; Smally, R. E. Science 1996, 273, 483-487.

(32) Rols, S.; Almairac, R.; Henrard, L.; Anglaret, E.; Sauvajol, J. L. Eur. Phys. J. B 1999, 10, 263-270.

(33) Cambedouzou, J.; Pichot, V.; Rols, S.; Launois, P.; Petit, P.; Klement, R.; Kataura, H.; Almairac, R. Eur. Phys. J. B 2004, 42, 31-45.

(34) Launois, P.; Chorro, M.; Verberck, B.; Albouy, P. A.; Rouzière, S.; Colson, D.; Forget, A.; Noé, L.; Kataura, H.; Monthioux, M.; Cambedouzou, J. Carbon 2010, 48, 89-98.

(35) Wang, J.; Zhu, Y.; Zhou, J.; Lu, X. H. Phys. Chem. Chem. Phys. 2004, 6, 829-835.

(36) Nakamura Y.; Ohno T. Materials Chemistry and Physics 2012, 132, 682-687 
